\title{
Erratum to: Development of novel multiplex microsatellite polymerase chain reactions to enable high-throughput population genetic studies of Schistosoma haematobium
}

\author{
B. L. Webster ${ }^{1,2^{*}}$, M. Rabone ${ }^{1}$, T. Pennance ${ }^{1,3}$, A. M. Emery ${ }^{1}$, F. Allan ${ }^{1}$, A. Gouvras ${ }^{1}$, S. Knopp ${ }^{1,4,5}$, A. Garba ${ }^{6}$, \\ A. A. Hamidou ${ }^{6}$, K. A. Mohammed ${ }^{7}$, S. M. Ame', D. Rollinson ${ }^{7}$ and J. P. Webster ${ }^{2,3}$
}

\footnotetext{
* Correspondence: b.webster@nhm. ac.uk

'Wolfson Wellcome Biomedical Laboratories, Department of Life Sciences, Natural History Museum, Cromwell Road, London SW7 5BD, UK ${ }^{2}$ Department of Infectious Disease Epidemiology, Imperial College Faculty of Medicine (St Mary's Campus), Norfolk Place, London W2 1PG, UK Full list of author information is available at the end of the article
}

Unfortunately, the original version of this article [1], contained a mistake. In Table 1, the primers for Sh6 and Sh9 were included incorrectly. Instead of GGGATGTATGCAGACTTG TTGTTTGGCTGCAGTAAC and GCTGAGCTTGAGATTG CTTCTGTCCCATCGATACC they should have been Sh6 Forward Primer GgTGGATtACGCAATAG, Sh6 Reverse Primer TTtAATCAACCGGGTGTC and Sh9 Forward Primer GGGATGTATGCAGACTTG, Sh9 Reverse Primer TTGTTTGGCTGCAGTAAC respectively.

A corrected version of Table 1 is included below.

\begin{abstract}
Author details
'Wolfson Wellcome Biomedical Laboratories, Department of Life Sciences, Natural History Museum, Cromwell Road, London SW7 5BD, UK. ${ }^{2}$ Department of Infectious Disease Epidemiology, Imperial College Faculty of Medicine (St Mary's Campus), Norfolk Place, London W2 1PG, UK. ${ }^{3}$ RVC Department of Pathology and Pathogen Biology, Centre for Emerging, Endemic and Exotic Diseases (CEEED), Royal Veterinary College, University of London, Hertsfordshire AL97TA, UK. ${ }^{4}$ Department of Epidemiology and Public Health, Swiss Tropical and Public Health Institute, Socinstrasse 57, Basel 4002, Switzerland. ${ }^{5}$ University of Basel, Petersplatz 1, Basel 4003, Switzerland. 'Réseau International Schistosomoses, Environnement, Aménagement et Lutte (RISEAL-Niger), 333,

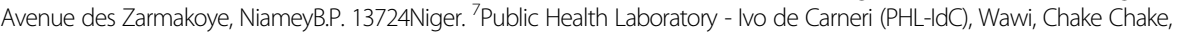
Pemba, United Republic of Tanzania.
\end{abstract}

Received: 1 October 2015 Accepted: 1 October 2015

Published online: 09 October 2015

\section{Reference}

1. Webster BL, Rabone M, Pennance T, Emery AM, Allan F, Gouvras S, et al. Development of novel multiplex microsatellite polymerase chain reactions to enable high-throughput population genetic studies of Schistosoma haematobium. Parasit and Vectors. 2015:8:432 
Table 1 Details of the 18 selected microsatellite loci and the characteristics of the two multiplex microsatellite PCR assays. Loci Sh1-15 are from Travis et al., 2013 and Loci C102, C111 and C131 are from Gower et al., 2011. For Niger $\boldsymbol{H o}=0.596, \boldsymbol{H e}=0.609$, for Pemba $\boldsymbol{H o}=0.599, \boldsymbol{H e}=0.638$. The overall $\boldsymbol{H o}=0.597, \mathbf{H e}=0.623$

\begin{tabular}{|c|c|c|c|c|c|c|c|c|c|c|c|}
\hline \multirow[t]{2}{*}{ Panel 1} & \multirow[t]{2}{*}{ Marker } & \multirow[t]{2}{*}{ Forward Primer 5'- 3' } & \multirow[t]{2}{*}{ Reverse Primer 5'- 3' } & \multirow[t]{2}{*}{ Dye } & \multirow{2}{*}{$\begin{array}{l}\text { Size Range } \\
\text { (bp) }\end{array}$} & \multirow[t]{2}{*}{ Repeat } & \multirow[t]{2}{*}{ A } & \multicolumn{2}{|c|}{ Niger } & \multicolumn{2}{|c|}{ Zanzibar } \\
\hline & & & & & & & & $H_{\circ}$ & $H_{e}$ & $H_{\circ}$ & $H_{\mathrm{e}}$ \\
\hline \multirow[t]{9}{*}{ Panel 1} & C102 & TGTCTCTGTGAATGACCGAAT & TTAGATGAATAATAATGTTGAAACCAC & VIC & 184-199 & $A \Pi T$ & 6 & 0.42 & 0.37 & 0.02 & 0.02 \\
\hline & Sh1 & GCATCCAATTTCGTACAC & CCACATTAGGCCAACAAG & VIC & $245-284$ & AAT & 13 & 0.76 & 0.72 & 0.84 & 0.80 \\
\hline & Sh14 & GTCCTCCTTCCCTCTTTG & CACATTCGTCCTAGATATCG & NED & $184-240$ & ACTC & 15 & 0.94 & 0.85 & 0.86 & 0.88 \\
\hline & C131 & CTTGTCATTTGGGCATTGTG & CATGGTGAGGTTCAAACGTG & NED & $253-265$ & AAT & 4 & 0.00 & 0.00 & 0.00 & 0.00 \\
\hline & Sh6 & GGTGGATTACGCAATAG & TTAAATCAACCGGGTGTC & NED & 309-321 & AAT & 7 & 0.48 & 0.44 & 0.84 & 0.76 \\
\hline & Sh9 & GGGATGTATGCAGACTTG & TTGTTGGCTGCAGTAAC & 6-FAM & $197-227$ & AAT & 11 & 0.46 & 0.76 & 0.46 & 0.86 \\
\hline & Sh3 & GCTGAGCTTGAGATTG & CTTCTGTCCCATCGATACC & 6-FAM & $270-366$ & AAT & 30 & 0.76 & 0.86 & 0.94 & 0.86 \\
\hline & C111 & СCCTTGTCTTCAATGCGTTA & GAACGTCTAACTGGCGATCA & PET & $201-225$ & ATT & 9 & 0.74 & 0.67 & 0.76 & 0.68 \\
\hline & Sh7 & TCCAAGCACCATTATCAAG & ACGGAAACTTGTTGAAATG & PET & 293-311 & AAT & 7 & 0.46 & 0.62 & 0.42 & 0.48 \\
\hline \multirow[t]{9}{*}{ Panel 2} & Sh2 & TTAGTGTGTTTGGCTTCAAC & CCTCGAATGAAATCCTGAC & NED & $155-218$ & AAT & 21 & 0.84 & 0.90 & 0.56 & 0.89 \\
\hline & Sh5 & TGTGCACAAGAAAGATTAAATG & ACGACAATGTTGCAAGTTC & NED & 263-314 & AAT & 16 & 0.78 & 0.81 & 0.36 & 0.48 \\
\hline & Sh13 & GAGCAGCTATTTCGTATCG & ACCGTGGACAGTTCATCAG & 6-FAM & $163-211$ & AAT & 17 & 0.78 & 0.72 & 0.68 & 0.64 \\
\hline & Sh4 & CCCATCGCTGATATTAAAG & TCTAGTCGTCTTGGGATCC & 6-FAM & $268-313$ & AAT & 13 & 0.84 & 0.78 & 0.72 & 0.79 \\
\hline & Sh10 & CGCATGTCATACCTATCTCC & GCTTATCAGGCCTATCTCC & PET & $183-207$ & AAT & 9 & 0.18 & 0.34 & 0.74 & 0.70 \\
\hline & Sh12 & CGTCTTAGTGAGCCAGATG & CTCGTGGACATCATCAG & PET & $245-278$ & AAC & 11 & 0.06 & 0.06 & 0.56 & 0.65 \\
\hline & Sh8 & CTAAACTGGCAAGATTTC & CAACGTGCCTTTATTTC & PET & $282-321$ & AAT & 14 & 0.76 & 0.81 & 0.84 & 0.83 \\
\hline & Sh11 & TTGGTITAGAAATTACATCACC & CCAACAATATTAATGGACAGC & VIC & $183-213$ & ATC & 9 & 0.68 & 0.58 & 0.68 & 0.69 \\
\hline & Sh15 & CTTCAGTAGGATTTGTTG & CGACGTCAAGCACTGTAC & VIC & $274-301$ & ATC & 10 & 0.78 & 0.65 & 0.50 & 0.466 \\
\hline
\end{tabular}

Panel $=$ single mulitplex PCR. A = observed number of alleles. Dye = the fluorescent dye label of the forward primer $(\mathrm{VIC}=$ green, NED = yellow, 6-FAM = Blue, PET = red). Ho = observed heterozygosity

$\boldsymbol{H e}=$ expected heterozygosity 